\title{
LETTER
}

doi:10.1017/S1041610213001312

\section{Personality disorders in older adults: expert opinion as a first step toward evaluating the criterion validity of an informant questionnaire (HAP)}

There is a lack of validated questionnaires for screening personality disorders (PDs) in older adults (e.g. Van Alphen et al., 2012). The development of measurement instruments is hampered because the criteria of DSM-IVTR PD are not age-neutral that might lead to over- and underdiagnosis (Balsis et al., 2007). As far as we know only three measurement instruments have been specifically developed for older adults, including the Hetero- Anamnestic Personality questionnaire (HAP; Barendse et al., 2013). However, we did not find any articles concerning the criterion validity of all ten PDs in an elderly population. In this Delphi study, a panel of experts examined two research questions: (1) To what extend are the items of the HAP age-neutral? (2) Does the HAP detect all ten specific PD's of DSM-IV-TR, based on qualitative research?

The Delphi technique is a research method whereby independent experts, after several rounds, reach consensus on the basis of each other's anonymous judgment of the subject. When empirical data are sparse the method can be used as first step to quantitative research (Van Alphen et al., 2012). The experts were five core members of the Dutch and Belgian research group Experts panel Personality \& Older Adults (EPO). The experts were researchers in the domain of PD in older adults and/or had many years of clinical experience in the field of PD in general and in older adults. The HAP is an informant questionnaire developed for use in geriatric care to assess premorbid personality characteristics. In previous research (Barendse et al., 2013), the psychometric properties of the HAP were examined in nursing home residents $(\mathrm{n}=$ $385)$ and elderly psychiatric patients $(\mathrm{n}=204)$ in the Netherlands and Belgium. The internal consistency of the 12 scales was good (AIC values varies from .023 to 0.53 ); the inter-rater and testretest reliability were good to excellent (intraclass correlations between 0.60 and 0.98 ); The construct validity, as operationalized through factor analyses, showed the same structure in both populations (coefficients of congruence between nursing home residents and elderly psychiatric patients with values of respectively $0.99,0.96$, and 0.98 for factors 1,2 , and 3). However, age-neutrality and the criterion validity of the HAP were not examined.

Therefore, the first author conducted the initial theoretical-substantive analysis and judged whether each item of the HAP corresponded in a semantic way to one or more of the criteria of the DSM-IVTR PD. Next, in a first round, the experts judged both the age neutrality of every HAP item and personal opinion of the first author regarding the way HAP items are related to DSM-PDs. In case of disagreement, the experts explained their decision. In the second and decisive round, the results and explanations of the participants were anonymously presented. Two rounds seemed to be enough for agreement in this expert panel. Agreement with a HAP item and the proposed DSM criterion was rated on a scale from 0 (full rejection) to 10 (full acceptance). A mean of $\geq 8$ was required to accept agreement about adherence of a HAP item to the DSM criterion. To rule out too much variation in opinions of the experts HAP items were not included if the standard deviation was above two.

The response rate was optimal; in both rounds $100 \%$. First, the five experts labeled all 62 items of the HAP as age neutral. Second, the HAP represented sufficient criteria for nine of the ten DSM-IV-TR PDs. More specific, the HAP represented all criteria of the antisocial, avoidant, and dependent PD. Besides, the HAP has just one missing Axis-II criterion regarding paranoid, narcissistic, obsessive-compulsive PD and three missing criteria regarding schizoid, borderline, and histrionic PD. Only schizotypal PD is not represented in the HAP (just two out of nine criteria had agreement with HAP items). A complete overview of the results is available from the first author on request. These initial results are promising; however, the potential of the HAP to screen for PDs in young and older adults should be further empirically explored. Complete polythetic criteria sets on the basis of HAP items can be made concerning antisocial, avoidant, and dependent PD and the criteria of most PDs can be presented largely. However, the HAP items clearly cannot be linked to sufficient criteria of schizotypal PD. Delphi research relies on level III evidence and cannot be more than a start of quantitative research (Van Alphen et al., 2012). The age neutrality of the HAP items is a hypothesis to be tested in follow-up research by for instance differential item functioning (DIF) analysis (e.g. Van den Broeck et al., 2012), 
followed by research regarding the criterion validity in (non) clinical samples of older adults.

\section{Conflict of interest}

None.

\section{References}

Balsis, S., Woods, C. M., Gleason, M. E. and Oltmanns, T. F. (2007). Overdiagnosis and underdiagnosis of personality disorders in older adults. American fournal of Geriatric Psychiatry, 15, 742-753.

Barendse, H. P. J., Thissen, A. J. C., Rossi, G., Oei, T. I. and Van Alphen, S. P. J. (2013). Psychometric properties of an informant personality questionnaire (the HAP) in a sample of older adults in the Netherlands and Belgium. Aging and Mental Health, 17, 623-629. doi: $10.1080 / 13607863.2012 .756458$.

Van Alphen, S. P. J. et al. (2012). Age related aspects and clinical implementations of diagnosis and treatment of personality disorders in older adults. Clinical Gerontologist, $1,27-41$.

Van Alphen, S. P. J., Sadavoy, J., Derksen, J. J. L. and Rosowsky, E. (2012). Editorial: features and challenges of personality disorders in late life. Aging and Mental Health, $16,805-810$.

Van den Broeck, J., Rossi, G., Dierckx, E. and De Clercq, B. (2012). Age-neutrality of the NEO-PI-R: potential differential item functioning in older versus younger adults. Fournal of Psychopathology and Behavioral Assessment, 34, 361-369. doi:10.1007/s10862-012-9287-4.

H.P.J. BARENDSE, ${ }^{1}$ G. RosSi ${ }^{2}$ AND S.P.J. VAN ALPHEN ${ }^{2,3}$

${ }^{1}$ Psychology Practice Barendse \& Thissen, Schijndel, the Netherlands

${ }^{2}$ Vrije Universiteit Brussel (VUB), Department of Clinical and Life Span Psychology, Brussels, Belgium

${ }^{3}$ Mondriaan Hospital, Department of Old Age Psychiatry, Heerlen-Maastricht, the Netherlands Email: barendse.hpj@home.nl 